\title{
Comparison of Fecal Collection Methods for Microbiome and Metabolomics Studies
}

\begin{abstract}
Zheng Wang ${ }^{1}$, Christine P. Zolnik ${ }^{2,3}$, Yunping Qiu ${ }^{4}$, Mykhaylo Usyk ${ }^{2}$, Tao Wang ${ }^{1}$, Howard D. Strickler ${ }^{1}$, Carmen R. Isasi ${ }^{1}$, Robert C. Kaplan ${ }^{1,5}$, Irwin J. Kurland ${ }^{4}$, Qibin Qi ${ }^{1 *}$ and Robert D. Burk ${ }^{1,2,6,7 *}$

${ }^{1}$ Department of Epidemiology and Population Health, Albert Einstein College of Medicine, Bronx, NY, United States, ${ }^{2}$ Department of Pediatrics, Albert Einstein College of Medicine, Bronx, NY, United States, ${ }^{3}$ Department of Biology, Long Island University, Brooklyn, NY, United States, ${ }^{4}$ Department of Medicine, Stable Isotope and Metabolomics Core Facility, Diabetes Center, Albert Einstein College of Medicine, Bronx, NY, United States, ${ }^{5}$ Public Health Sciences Division, Fred Hutchinson Cancer Research Center, Seattle, WA, United States, ${ }^{6}$ Microbiology and Immunology, Albert Einstein College of Medicine, Bronx, NY, United States, ' Obstetrics, Gynecology and Women's Health, Albert Einstein College of Medicine, Bronx, NY, United States
\end{abstract}

Background: Integrated microbiome and metabolomics analyses hold the potential to reveal interactions between host and microbiota in relation to disease risks. However, there are few studies evaluating how field methods influence fecal microbiome characterization and metabolomics profiling.

Methods: Five fecal collection methods [immediate freezing at $-20^{\circ} \mathrm{C}$ without preservative, OMNIgene GUT, 95\% ethanol, RNAlater, and Flinders Technology Associates (FTA) cards] were used to collect 40 fecal samples from eight healthy volunteers. We performed gut microbiota $16 \mathrm{~S}$ rRNA sequencing, untargeted metabolomics profiling, and targeted metabolomics focusing on short chained fatty acids (SCFAs). Metrics included $\alpha$-diversity and $\beta$-diversity as well as distributions of predominant phyla. To evaluate the concordance with the "gold standard" immediate freezing, the intraclass correlation coefficients (ICCs) for alternate fecal collection systems were calculated. Correlations between SCFAs and gut microbiota were also examined.

Results: The FTA cards had the highest ICCs compared to the immediate freezing method for $\alpha$-diversity indices (ICCs $=0.96,0.96,0.76$ for Shannon index, Simpson's Index, Chao-1 Index, respectively), followed by OMNIgene GUT, RNAlater, and 95\% ethanol. High ICCs (all >0.88) were observed for all methods for the $\beta$-diversity metric. For untargeted metabolomics, in comparison to immediate freezing which detected 621 metabolites at $\geq 75 \%$ detectability level, 95\% ethanol showed the largest overlapping set of metabolites ( $n=430 ; 69.2 \%$ ), followed by FTA cards ( $n$ $=330 ; 53.1 \%)$ and OMNIgene GUT $(n=213$; 34.3\%). Both OMNIgene GUT (ICCs $=0.82,0.93,0.64)$ and FTA cards (ICCs $=0.87,0.85,0.54)$ had acceptable ICCs for the top three predominant SCFAs (butyric acid, propionic acid and acetic acid). Nominally significant correlations between bacterial genera and SCFAs $(P<0.05)$ were observed in fecal samples collected by different methods. Of note, a high correlation between the genus Blautia (known butyrate producer) and butyric acid was observed for both immediate freezing $(r=0.83)$ and FTA cards $(r=0.74)$. 


\begin{abstract}
Conclusions: Four alternative fecal collection methods are generally comparable with immediate freezing, but there are differences in certain measures of the gut microbiome and fecal metabolome across methods. Choice of method depends on the research interests, simplicity of fecal collection procedures and ease of transportation to the lab, especially for large epidemiological studies.
\end{abstract}

Keywords: fecal microbiome, metabolomics, sampling methods, multi-omics integration, integrative analysis

\section{INTRODUCTION}

Over the past decade, the microbiota's potential impact on human chronic diseases has garnered increasing interest. Many health conditions, such as diabetes, cardiovascular diseases, and cancers, were found to be associated with microbiota, particularly with the gut microbiota (Ley, 2010; Kostic et al., 2014; Forslund et al., 2015; Vogtmann and Goedert, 2016; Chong and Xia, 2017). Interactions between host and microbiota occur primarily through evolutionarily conserved chemical dialogs that involve a multitude of metabolites and pathways (Martin et al., 2009; Candela et al., 2011). Thus, integrating analyses of microbiome and related metabolites should facilitate understanding the role of microbiota in human chronic diseases (Fiehn, 2002; Patti et al., 2012; Chong and Xia, 2017).

The measurements of the gut microbiota are influenced by numerous factors, including "wet-lab" protocols used for assaying specimens, and "dry-lab" approaches used in data processing. In addition, the field methods used for fecal sample collection comprise another critical methodological feature that is often difficult or impossible to remediate once a protocol has been fielded (Cardona et al., 2012; Choo et al., 2015; Sinha et al., 2015, 2016). A number of fecal collection methods, such as $95 \%$ ethanol, OMINIgene GUT Kit, RNAlater Stabilization Solution, fecal occult blood test (FOBT) cards, and fecal immunochemical test tubes, have been examined for gut microbiota profiling, and compared to immediate freezing at $-20^{\circ} \mathrm{C}$, which is considered as the "gold standard" (Nechvatal et al., 2008; Flores et al., 2015; Voigt et al., 2015; Sinha et al., 2016; Song et al., 2016; Vogtmann et al., 2017b). These collection systems showed relatively high reproducibility and stability at ambient temperature. Nevertheless, variations were observed across different methods (Nechvatal et al., 2008; Flores et al., 2015; Voigt et al., 2015; Sinha et al., 2016; Song et al., 2016; Vogtmann et al., 2017b). However, it is unclear if specific collection methods are adequate for integrated microbiome and metabolomics studies. A recent study compared the aforementioned fecal collection methods for untargeted metabolomics profiling, and the 95\% ethanol method showed the highest concordance with the "gold standard" (Loftfield et al., 2016). To the best of our knowledge, no studies have compared fecal collection methods for both gut microbiota profiling and fecal metabolomics simultaneously.

In order to determine the optimal collection method for integrated microbiome and metabolomics studies, we compared five fecal collection methods for gut microbiota 16S rRNA gene $\mathrm{V} 4$ region sequencing and metabolomics profiling. FTA cards, which make use of a swab sample that is smeared on a card and then allowed to dry, use a nucleic acid stabilizer and are similar in format to FOBT cards (Vandeputte et al., 2017). This supports the rationale for FTA cards as effective tools which may provide comparable microbiota diversity results compared with immediate freezing (Song et al., 2016; Wong et al., 2017),without the need for long term cold storage. In addition to untargeted metabolomics profiling, we also designed a targeted metabolomics panel focusing on short chained fatty acids (SCFAs), which are major metabolites produced by gut microbiota. This part of the study entailed measurements, across different fecal collection methods, of fecal SCFA concentrations as well as correlations between SCFAs and gut microbiota.

\section{MATERIALS AND METHODS}

\section{Fecal Specimen Collection}

Five different methods (OMNIgene GUT (DNA Genotek, Ottawa, Canada), 95\% ethanol, RNAlater (Invitrogen, Carlsbad, CA), FTA cards (GE Healthcare, Chicago, IL), and immediate freezing at $-20^{\circ} \mathrm{C}$ were used to collect 40 fecal samples from eight healthy volunteers. The fecal samples were self-collected using a disposable paper inverted hat (Protocult collection device, ABC Medical Enterprises, Inc., Rochester, MN). Each participant collected five fecal samples in total from a single specimen, including one sample for each of the five different methods. The participants sampled the stool with a plastic applicator, spreading a small amount on a Whatman FTA card, as the first sample. They collected a second sample which was placed in a supplied container including a stabilizer (RNAlater) and $0.5 \mathrm{~mm}$ diameter glass beads and instructed to shake the tube in order to mix the stool and the preservative which stabilizes DNA and RNA (Flores et al., 2012). The third sample was placed in the OMNIgene GUT tube, using the supplied applicator and following the manufacturer's directions. The fourth sample was placed in a supplied tube containing 95\% ethanol and glass beads. All samples collected by the aforementioned four methods were left at room temperature. The fifth sample was placed in a supplied container with no solution, placed in a plastic bag and put into a household freezer immediately. Within $24 \mathrm{~h}$, all samples were delivered to the laboratory using a styrofoam box containing ice packs. Upon arrival at the lab, two aliquots of each fecal sample were immediately created for the gut microbiome and metabolomics analyses and stored at $-80^{\circ} \mathrm{C}$.

The study was reviewed and approved by the institutional review board (IRB) at the Albert Einstein College of Medicine. Written informed consent was obtained from all participants. 


\section{DNA Extraction and 16S rRNA Gene V4 Region Sequencing}

Laboratory procedures were conducted under a hood (AirClean Systems, Creedmoor, NC) to limit environmental contamination. Total DNA was extracted from stool samples with the PowerLyzer PowerSoil DNA Isolation Kit (MO BIO laboratories Inc., Carlsbad, CA), following the manufacturer's procedures. Briefly, a $100 \mu \mathrm{l}$ homogenized aliquot from the RNAlater, 95\% ethanol and OMNIgene GUT tube samples, a small scoopful of the immediate freezing sample and 1/4 of the FTA card spot were each added to PowerLyzer bead tubes with $60 \mu 1$ of Solution C1 (vortexed to mix) and beaten with a FastPrep-24 homogenizer (MP Biomedicals, Santa Ana, CA) at speed 6.0 for $40 \mathrm{~s}$. The samples were centrifuged at $10,000 \mathrm{~g}$ for $30 \mathrm{~s}$ and the supernatant was removed and placed in a new collection tube. The DNA was isolated by column purification and collected in $100 \mu \mathrm{l}$ of elution buffer (Solution C6).

PCR amplification of the V4 hypervariable region of the $16 \mathrm{~S}$ rRNA gene was performed using primers 16SV4_515F and 16SV4_806R (Caporaso et al., 2012) each with 12-bp unique Golay barcodes, resulting in unique dual barcodes for each forward and reverse primer pair. PCR reactions were performed with $16.25 \mu \mathrm{l}$ of nuclease-free PCR-grade water, $2.5 \mu \mathrm{l}$ of 10X Buffer w/ $\mathrm{MgCl}_{2}$ (Affymetrix,Santa Clara, CA), $1 \mu \mathrm{l}$ of $\mathrm{MgCl}_{2}$ (25 mM, Affymetrix), $0.5 \mu \mathrm{l}$ of dNTPs (10 mM, Roche, Pleasanton, CA), $0.25 \mu$ l of AmpliTaq Gold DNA Polymerase (5 $\mathrm{U} / \mu \mathrm{l}$, Applied Biostystems, Foster City, CA), $0.5 \mu \mathrm{l}$ of HotStartIT FideliTaq (2.5 U/ $\mu$ l, Affymetrix), $1 \mu$ l of each primer $(5 \mu \mathrm{M})$, and $2 \mu \mathrm{l}$ of extracted DNA. Thermal cycling conditions included an initial denaturation at $95^{\circ} \mathrm{C}$ for $5 \mathrm{~min}$; followed by 15 cycles at $95^{\circ} \mathrm{C}$ for $1 \mathrm{~min}, 55^{\circ} \mathrm{C}$ for $1 \mathrm{~min}$, and $68^{\circ} \mathrm{C}$ for $1 \mathrm{~min}$; followed by 15 cycles at $95^{\circ} \mathrm{C}$ for $1 \mathrm{~min}, 60^{\circ} \mathrm{C}$ for $1 \mathrm{~min}$, and $68^{\circ} \mathrm{C}$ for $1 \mathrm{~min}$; and a final extension for $10 \mathrm{~min}$ at $68^{\circ} \mathrm{C}$ on a GeneAmp PCR System 9700 (Applied Biosystems).

Dual indexed PCR products were isolated and combined and $100 \mu \mathrm{l}$ of the pooled products were run on a $4 \%$ agarose gel at $80 \mathrm{~V}$ for $2 \mathrm{~h}$. The bands ( $\sim 450 \mathrm{bp}$ ) were excised from the agarose gel and purified using a QIAquick Gel Extraction Kit (QIAGEN, Valencia, CA) and eluted in $30 \mu \mathrm{l}$ of elution buffer. The purified PCR products were quantified using a Qubit 2.0 Fluorometic High Sensitivity dsDNA Assay (Life Technologies, Carlsbad, CA).

A sequencing library was prepared using KAPA LTP Library Preparation Kit (Roche Sequencing Solutions, Pleasanton, CA) according to the manufacture's protocol. The size integrity of the amplicon was validated with a 2100 Bioanalyzer (Agilent Technologies, Santa Clara, CA) at the Albert Einstein College of Medicine Genomics Core. High-throughput amplicon sequencing was conducted on a MiSeq (Illumina, San Diego, CA) using $2 \times 300$ paired-end fragment reads at the Albert Einstein College of Medicine Sequencing Core.

\section{Microbiome Bioinformatics Analysis}

Illumina reads were quality trimmed to remove bases with PHRED quality scores below 25 using prinseq version 0.20.4 (Schmieder and Edwards, 2011). Samples were then demultiplexed based on the sample specific dual Golay barcode combinations. To generate a full length $16 \mathrm{~S}$ rRNA V4 region sequence, the paired-end reads were joined into a single sequence with the FLASH algorithm (Magoc and Salzberg, 2011). After quality control, one sample was excluded in the analysis due to unexpected low reads $(<1,000)$. The average coverage was $\sim$ 18,000 reads per sample.

Microbiome bioinformatics analysis was performed using the Quantitative Insights Into Microbial Ecology (QIIME) software package, version 1.9 (Caporaso et al., 2010b). Sequences were clustered into operational taxonomic units (OTUs) based on $\geq 97 \%$ similarity by the UCLUST algorithm. Phylogenetic reconstruction was performed by first aligning OTU representative sequences using PyNAST (Caporaso et al., 2010a). Taxonomy was then assigned using the QIIME closed reference OTU picking method with the Greengenes database, version 13.5 (DeSantis et al., 2006; McDonald et al., 2012). We calculated the relative abundance from phylum level to genus level for each collection method. $\alpha$-diversity indices (Shannon index, Simpson's Index, and Chao 1 index) and $\beta$ - diversity BrayCurtis distances were calculated using the $\mathrm{R}$ phyloseq/vegan package (McMurdie and Holmes, 2013; Oksanen, 2015).

\section{Metabolomics Profiling}

Sample extraction and derivatization for untargeted profiling: Metabolites extraction was performed with methanol (ethanol for ethanol collected samples): water $=3: 1$. The solutions in OMINIgene GUT kit and RNAlater were considered as water. After vortex and sonication for $5 \mathrm{~min}$, the samples were centrifuged at $15,000 \mathrm{rpm}$ for $15 \mathrm{~min}$. A volume of $500 \mu \mathrm{l}$ of supernatant was added with internal standards $(10 \mathrm{ul}$ of $10 \mathrm{nmol}$ $\mathrm{U}^{13} \mathrm{C}$ citrate and $5 \mathrm{nmol}$ of $\mathrm{U}^{13} \mathrm{C}$ succinate) and dried under gentle nitrogen flow. Samples collected with RNAlater were not dried after the overnight drying, and were not used for the next step.

Dried samples from ethanol, immediate freezing, FTA cards, and Omni kit were subjected to a two-step derivatization (methoximation and silylation) as described previously (Qiu et al., 2009). The samples were analyzed by gas chromatography mass spectrometry (GC-MS, Agilent, USA) with a 30-meter DB-5MS column. The oven program was initiated with $60^{\circ} \mathrm{C}$ for $1 \mathrm{~min}$, and increased to $320^{\circ} \mathrm{C}$ and kept for $5 \mathrm{~min}$. A full scan mode was used with the mass range of 35-600 Da. Raw data was analyzed in Genedata Expressionist (Genedata, Basel, Switzerland) software. Metabolite annotation was performed by comparing the mass spectrum and retention time to our inhouse libraries and commercially available libraries (i.e., Fiehn and NIST).

Targeted short chain fatty acids analysis: Feces samples collected with 4 methods (immediate freezing, OMINIgene GUT, RNAlater, and FTA cards) were used for SCFA analysis with propyl chloroformate (PCF) derivatization. Due to interference of ethanol with propanol, samples collected with ethanol were not used for PCF analysis (ethanol will react with SCFAs to form ethyl ester under this derivatization condition).

A volume of $1 \mathrm{~mL}$ water was added to dry samples (immediate freezing and FTA cards). All samples were vortexed for $2 \mathrm{~min}$, and sonicated for $5 \mathrm{~min}$. The supernatant was collected after 
centrifugation of $15 \mathrm{~min}$ at $15,000 \mathrm{rpm}$. A volume of 500 $\mu l$ of supernatant was transferred into a new glass tube for derivatization with $10 \mu \mathrm{l}$ of internal standards $(500 \mathrm{ug} / \mathrm{ml}$ propanoic acid_D5, and $100 \mu \mathrm{g} / \mathrm{mL}$ butyric acid_D7). The derivatization and GC-MS analysis followed our previous protocol (Zheng et al., 2013). Chemstation was used for data analysis.

\section{Statistical Analysis}

Kruskal-Wallis test was applied to compare the differences in the microbial $\alpha$-diversity indices (Shannon index, Simpson index and Chao-1 index) across five fecal collection methods. PERMANOVA with Bray-Curtis dissimilarity and principalcoordinate analysis (PCoA) were carried out for the microbial $\beta$ diversity analyses. To evaluate the concordance of different fecal collection methods compared with the gold standard immediate freezing, we calculated the intraclass correlation coefficients (ICCs) for the three $\alpha$-diversity indices, the $\beta$-diversity metric Bray-Curtis distances, and the relative abundances of the top three dominant phyla (Actinobacteria, Bacteroidetes, Firmicutes). We calculated the distance-based ICC for $\beta$-diversity and the $95 \%$ CIs using 1,000 bootstrap value according to the algorithm described elsewhere (Vogtmann et al., 2017a). Square-root transformation was conducted for the relative abundance of taxonomic units before analysis (Nakatsu et al., 2015). For untargeted metabolomics, metabolite values were first normalized using Quantile normalization (Bolstad et al., 2003) for each method and then $\log 10$ transformation was performed before analyses. Missing values were imputed with $1 / 2$ minimum values for a given metabolite within one method. ICCs between collection methods for metabolites with $\geq 75 \%$ detectability (e.g., measured in $\geq 6$ out of 8 participants) were calculated. For targeted metabolomics, ICCs between collection methods for eight SCFAs with $\geq 75 \%$ detectability were calculated. In addition, Spearman correlations between microbial genera and SCFAs were computed within each method. $\mathrm{R}$ packages vegan, icc, DESeq2, and phyloseq were used for the statistical analyses (Anders and Huber, 2010; McMurdie and Holmes, 2013; Love et al., 2014; Oksanen, 2015).

\section{Accession Number}

The sequencing data are available at the Sequence Read Archive (SRA) under accession number SRP153121.

\section{RESULTS}

\section{Microbiome Analyses}

There were no significant differences in $\alpha$-diversity indices (Shannon index, Simpson's Index, Chao-1 Index) among the five fecal collection methods (all $P>0.05$ ). The $\beta$ - diversity Bray Curtis distance PCoA analysis indicated that interindividual differences were responsible for the majority the microbial communities' variability, while very small differences were observed across different collection methods within samples (Figure 1). The FTA cards demonstrated the highest concordance with immediate freezing for three $\alpha$-diversity indices (ICCs $=0.96,0.96,0.76$ for Shannon index, Simpson's
Index, Chao-1 Index, respectively), followed by the OMNIgene GUT (ICCs $=0.94,0.97,0.51)$, RNAlater $($ ICCs $=0.75,0.79$, 0.51 ), and $95 \%$ ethanol (ICCs $=0.25,0.36,0.01$ ) (Figure 2A). For the $\beta$-diversity metric, high ICCs (all $>0.88$ ) were observed for all four methods compared to immediate freezing.

Concordance results of the three pre-dominant phyla across collection methods are shown in Figure 2B. For Actinobacteria, the FTA cards $($ ICC $=0.97)$ and $95 \%$ ethanol $($ ICC $=0.97)$ showed the highest concordance with immediate freezing. OMNIgene GUT had nearly as high an ICC for Actinobacteria (0.95), while RNAlater had a slightly lower ICC of 0.84 . For Firmicutes, the FTA cards (ICC $=0.91$ ) showed the highest concordance with immediate freezing, slightly exceeding the ICC for OMNIgene GUT (0.87), and substantially superior to RNAlater (ICC $=0.64$ ) and $95 \%$ ethanol (ICC $=0.34$ ). For Bacteroidetes, OMNIgene GUT had the highest ICC although this only achieved a moderate concordance value of ICC $=0.76$, where as the other samples showed relatively poor concordance with Immediate freezing. The taxonomic analysis at genus level across five collection methods is shown in Supplementary Figure S1.

\section{Metabolomics: Untargeted Metabolites}

Using untargeted metabolomics, the number of metabolites detected in at least one fecal sample was 747 with immediate freezing, 694 with OMNIgene GUT, 742 with $95 \%$ ethanol, and 708 with FTA cards. Our attempts to measure metabolites in samples preserved with RNAlater failed. We compared the four other methods at multiple levels, by further restricting to the subset of metabolites with $\geq 50, \geq 75$ and $100 \%$ detectability (Table 1). When we limited the data to the subset of metabolites that had $\geq 75 \%$ detectability, there were $621,245,467$, and 376 metabolites in fecal samples collected with immediate freezing, OMNIgene GUT, 95\% ethanol, and FTA cards, respectively. Compared with the gold standard immediate freezing which had 621 metabolites with $75 \%$ detectability in fecal samples, $95 \%$ ethanol showed the largest number of identical metabolites $(n=$ 430; 69.2\%). The next-best overlap of detected metabolites was observed for FTA cards ( $n=330 ; 53.1 \%)$, followed by OMNIgene GUT $(n=231 ; 34.3 \%)$.

The next concordance analyses were limited to the subset of metabolites with $\geq 75 \%$ detectability between the gold standard and each of the other collection methods (i.e., those metabolites above the $\geq 75 \%$ detection limit were included). The median of the ICCs (interquartile range; IQR) were $0.32(0.12-0.63)$ for OMNIgene GUT, $0.27(0.13-0.60)$ for $95 \%$ ethanol, and 0.27 (0.12-0.63) for FTA cards (Figure 3A).

Among annotated metabolites, the number detected in at least one fecal sample was 131 for immediate freezing, 128 for OMNIgene GUT, 132 for $95 \%$ ethanol, and 128 for FTA cards (Table 1). When the analysis was further restricted to the subset of annotated metabolites detected in $\geq 75 \%$ of samples, there were $123,74,118$, and 97 metabolites in fecal samples collected with immediate freezing, OMNIgene GUT, 95\% ethanol, and FTA cards, respectively (Table 1 and Supplementary Figure S2). Compared with immediate freezing which had 123 annotated metabolites with $\geq 75 \%$ detectability, $95 \%$ ethanol showed the 

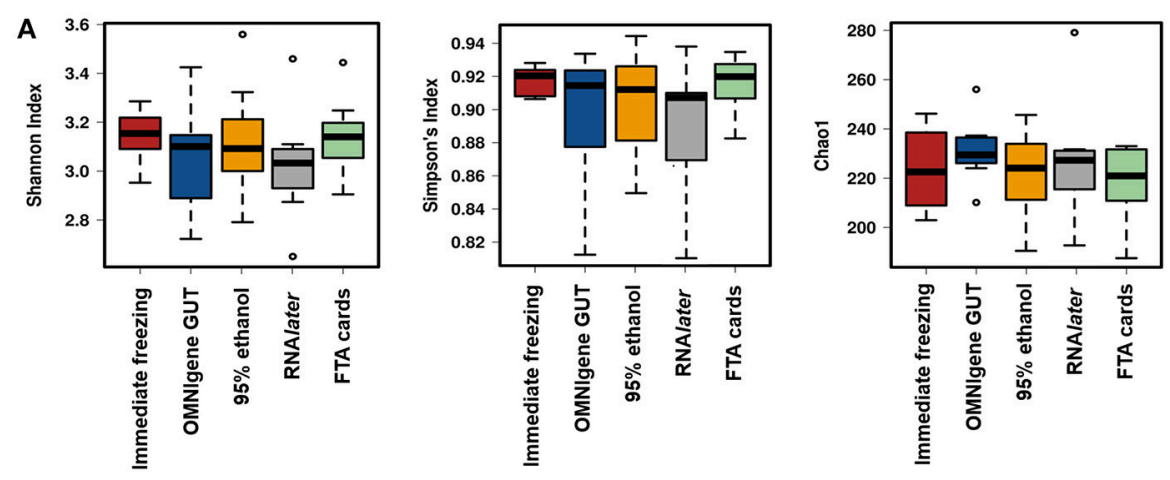

B
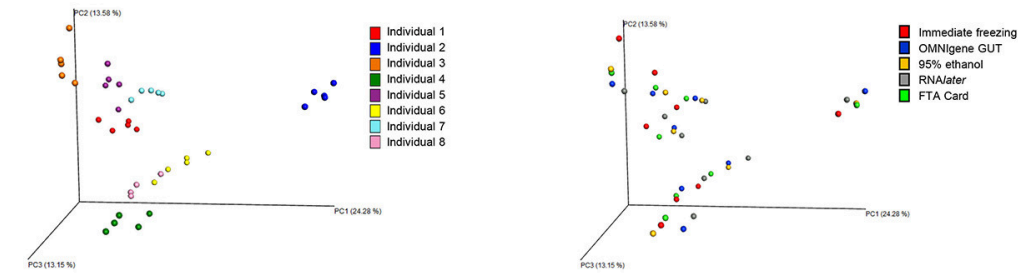

FIGURE 1 | (A) $\alpha$ - diversity analyses for the five fecal collection methods. (B) Principal coordinates analyses (PCoAs) of $\beta$ - diversity using Bray-Curtis distances.
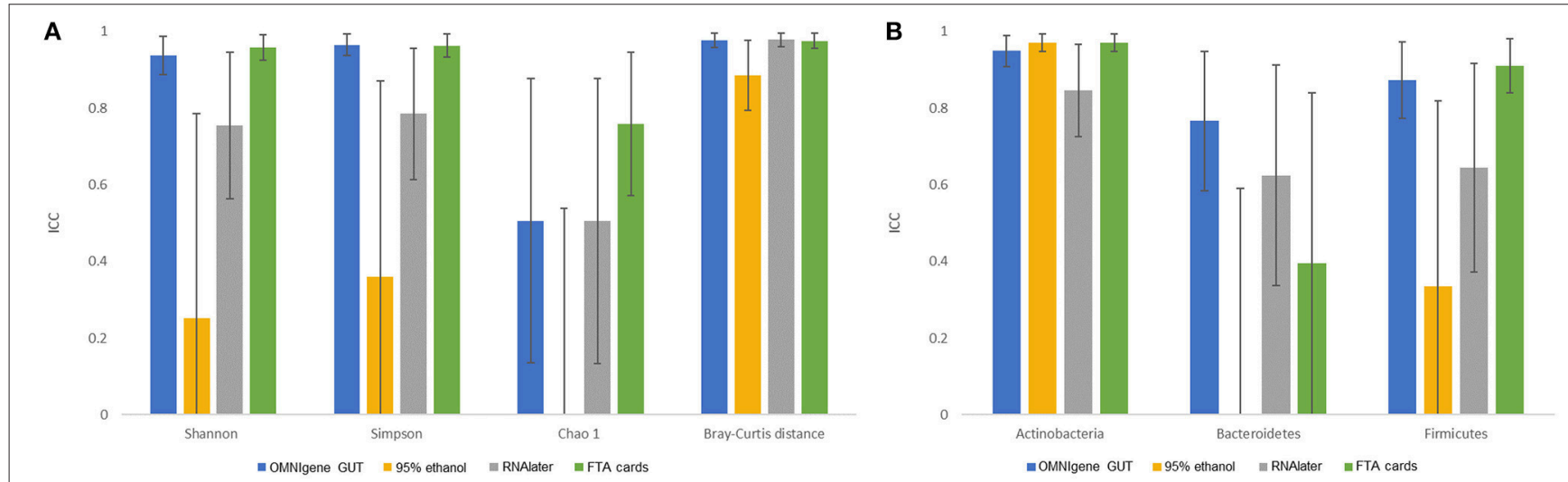

FIGURE 2 | The concordance of microbiota obtained by different fecal collection methods compared with the gold standard immediate freezing: intraclass correlation coefficients (ICC) and 95\% Cl for: (A) three $\alpha$ - diversity metrics and $\beta$ - diversity Bray-Curtis distance. (B) top three phyla.

largest number of identical metabolites ( $n=112 ; 91.1 \%$ ), followed by FTA cards ( $n=95 ; 77.2 \%)$, and OMNIgene GUT $(n=72 ; 58.5 \%)$. Despite high to moderate concordance across methods in terms of the numbers of metabolites detected, the reproducibility (ICC) of measurements across methods was relatively poor. The median (IQR) of the ICCs for identical metabolites at $75 \%$ detectability level were $0.21(0.12-0.61)$ for OMNIgene GUT, $0.35(0.13-0.64)$ for $95 \%$ ethanol, and 0.26 (0.13-0.57) for FTA cards (Figure 3B).

\section{Metabolomic Analyses: SCFAs}

In total, 10 targeted SCFAs were detected in fecal samples collected with immediate freezing, OMNIgene GUT and FTA cards (Supplementary Table S1). SCFAs could not be detected in samples preserved in RNAlater. All three collection methods had $100 \%$ detectability for the three predominant SCFAs: butyric acid, propionic acid, and acetic acid. For an additional set of seven measured SCFAs, detectability was $100 \%$ for immediate freezing, and detectability varied for OMNIgene GUT and FTA cards (Supplementary Table S1).

We then evaluated concordance of three predominant and seven other non-predominant SCFAs with $\geq 75 \%$ detectability between gold standard and the other methods (Figure 4). The ICCs for butyric acid were 0.82 (95\% confidence interval [CI], 0.68 to 0.97 ) for OMNIgene GUT, 0.87 (95\% CI, 0.74-0.97) for FTA cards. The ICCs for propionic acid were 0.93 (95\% CI, 0.87-0.99) for OMNIgene GUT, and 0.85 (95\% CI, 0.74-0.97) for FTA cards. The ICCs of Acetic acid were relatively lower for OMNIgene GUT $(0.64,95 \%$ CI, 0.34-0.93) and FTA cards $(0.54,95 \%$ CI, 0.16-0.91). For five non-predominant SCFAs, both 
TABLE 1 | Comparison of the number of metabolites at multiple detectability levels across different collection methods.

\begin{tabular}{|c|c|c|c|c|c|}
\hline $\begin{array}{l}\text { Detectability level of } \\
\text { metabolites }\end{array}$ & Methods & $\begin{array}{l}\text { Total number of } \\
\text { metabolites }\end{array}$ & $\begin{array}{c}\text { Number of metabolites } \\
\text { shared by GS and method } \\
\text { ( } \% \text { of GS) }\end{array}$ & $\begin{array}{c}\text { Number of known } \\
\text { metabolites }\end{array}$ & $\begin{array}{c}\text { Number of known metabolites } \\
\text { shared by GS and method (\% of GS } \\
\text { known metabolites) }\end{array}$ \\
\hline \multirow[t]{4}{*}{ All metabolites } & Immediate freezing & 747 & - & 131 & - \\
\hline & OMNIgene GUT & 694 & 694 (92.9\%) & 128 & 128 (97.7\%) \\
\hline & $95 \%$ ethanol & 742 & 741 (99.2\%) & 132 & 131 (100\%) \\
\hline & FTA cards & 708 & 707 (94.7\%) & 128 & 127 (97.0\%) \\
\hline \multirow[t]{4}{*}{$\geq 50 \%$ detectability } & Immediate freezing & 705 & & 129 & - \\
\hline & OMNIgene GUT & 432 & 409 (58.0\%) & 106 & 105 (81.4\%) \\
\hline & $95 \%$ ethanol & 638 & $613(87.0 \%)$ & 126 & 124 (96.1\%) \\
\hline & FTA cards & 518 & 494 (70.1\%) & 119 & 117 (90.7\%) \\
\hline \multirow[t]{4}{*}{$\geq 75 \%$ detectability } & Immediate freezing & 621 & - & 123 & - \\
\hline & OMNIgene GUT & 245 & $213(34.3 \%)$ & 74 & $72(58.5 \%)$ \\
\hline & $95 \%$ ethanol & 467 & $430(69.2 \%)$ & 118 & 112 (91.1\%) \\
\hline & FTA cards & 376 & 330 (53.1\%) & 97 & 95 (77.2\%) \\
\hline \multirow[t]{4}{*}{ 100\% detectability } & Immediate freezing & 393 & - & 104 & - \\
\hline & OMNlgene GUT & 73 & $51(13.0 \%)$ & 29 & 27 (26.0\%) \\
\hline & $95 \%$ ethanol & 332 & 255 (65.9\%) & 97 & $88(84.6 \%)$ \\
\hline & FTA cards & 192 & 140 (35.6\%) & 70 & $64(61.5 \%)$ \\
\hline
\end{tabular}

GS, gold standard.

methods showed very high concordance for isovaleric acid and hexanoate, but relatively lower concordance for isovaleric acid, valeric acid and 2-methylbutyric acid compared to immediate freezing (Supplementary Table S1).

\section{Bacterial Populations vs. SCFA Levels}

We then explored the correlations between bacterial genera and the three predominant SCFAs in fecal samples. Nominally significant correlations between bacterial genera and SCFAs were observed. In immediate freezing fecal samples, we found nine correlations between bacterial genera and SCFAs $(P<0.05)$ (Table 2). The same trends for most correlations were observed in fecal samples collected with OMNIgene GUT and FTA cards, and some correlations were highly consistent across the three methods. For example, the correlation coefficients between Blautia and butyric acid were $0.83(P=0.01), 0.62(P=0.10)$, and $0.74(P=0.037)$ for immediate freezing, OMNIgene GUT and FTA cards, respectively. In addition, nominally significant correlations between the family Rikenellaceae and butyric acid, and between the genus Faecalibacterium and propionic acid were observed for FTA cards; and nominally significant correlations between the family Clostridiales and butyric acid, and between Rikenellaceae and butyric acid were observed for OMNIgene GUT.

\section{DISCUSSION}

The immediate freezing without preservative has been widely used as a gold standard for gut microbiome analyses, as this method preserves microbial composition similar to analysis of a fresh sample and also avoids potential influence of added preservative (Flores et al., 2015; Loftfield et al., 2016; Song et al., 2016; Vogtmann et al., 2017b). In the current report, our data indicate that compared with the immediate freezing method, alternative specimen collection systems not requiring freezing including OMINIgene GUT, 95\% ethanol, RNAlater, and FTA cards all can be informative for gut microbiome analysis (Song et al., 2016; Vogtmann et al., 2017b). All four methods showed relatively high concordance with the immediate freezing method for the $\beta$-diversity metric. Furthermore, there were small differences in microbial $\beta$-diversity Bray-Curtis distances across methods, while inter-individual differences were responsible for the majority of variation in microbial $\beta$-diversity. However, concordance for microbial $\alpha$-diversity varied across different methods. The FTA cards and OMNIgene GUT showed relatively higher concordance with the immediate freezing for three $\alpha$-diversity indices, followed by RNAlater and 95\% ethanol. Consistently, 95\% ethanol was reported to have relatively low validity for $\alpha$-diversity indices (Vogtmann et al., 2017b).

Interestingly, concordance for predominant microbial phyla varied across different methods as well as microbial phyla. For example, FTA cards had high ICCs $(\geq 0.85)$ for Actinobateria and Firmicutes but not for Bacteroidetes $(\mathrm{ICC}=0.40)$. Concordances of all four methods with the gold standard were generally high for the Actinobateria, but low for Bacteroidetes. Our results are supported by previous data (Vogtmann et al., 2017a), and previous studies that have indicated that some taxa are more sensitive to changes in collection and storage conditions (i.e., among immediate freezing, RNAlater and TE buffer) (Choo et al., 2015). RNAlater was reported to have decreased DNA purity which could interfere with downstream analyses (Dominianni et al., 2014). In addition, the cellular composition of Gram-positive and Gram-negative bacteria could be different, which presumably leads to differences in the ability to preserve DNA in some collection methods (Bahl et al., 2012; Fouhy et al., 2015). In our study, better concordances 


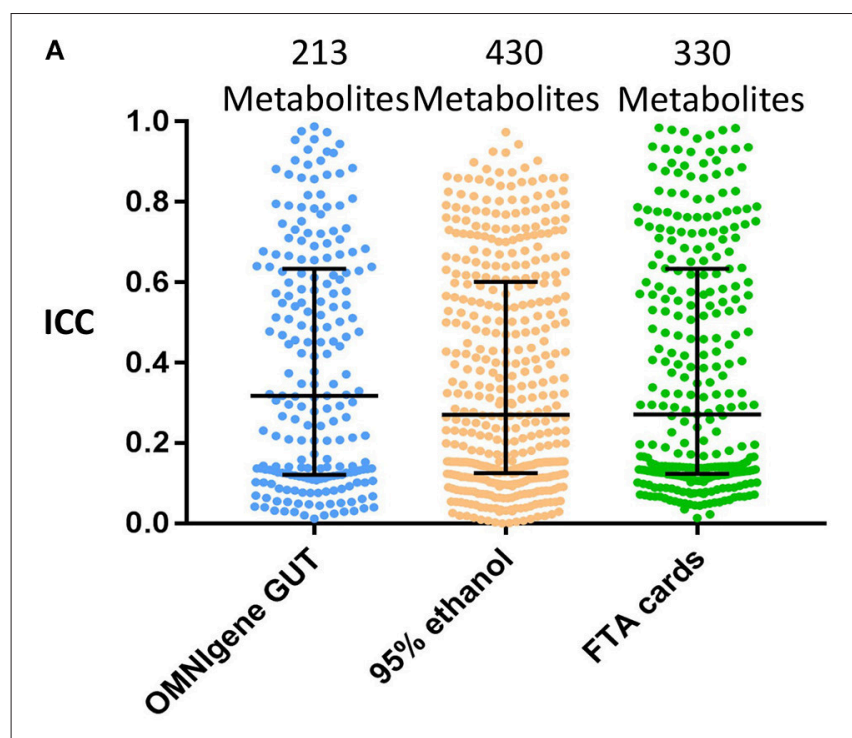

B
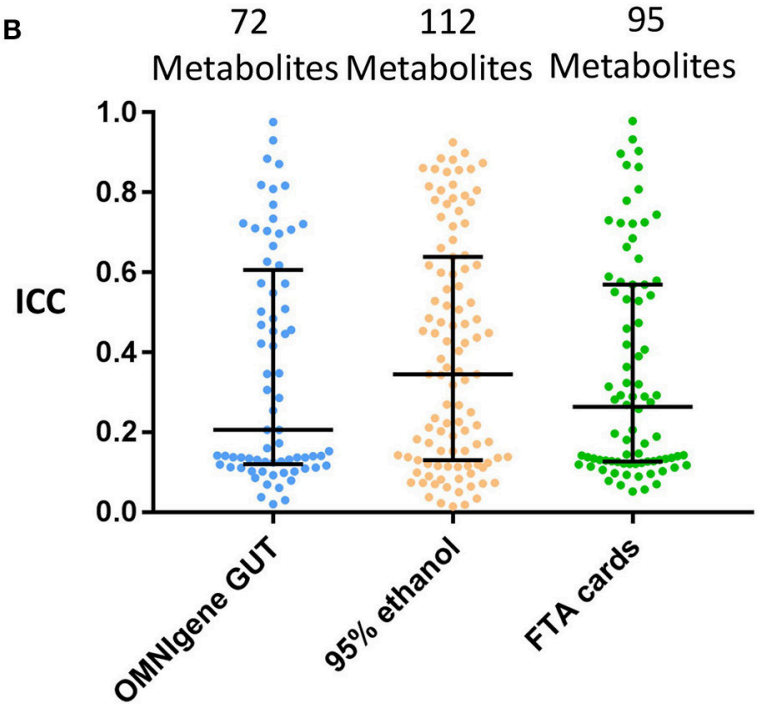

FIGURE 3 | The concordance of metabolite detection by different fecal collection methods. Stool samples collected by the indicated methods were compared with the gold standard of immediate freezing for metabolite detection estimated the ICCs at $\geq 75 \%$ metabolites detectability level. Log10 transformation and Quantile normalization were used. Missing values were imputed with $1 / 2$ minimum value for a given metabolite within one method. Highlighted medians and IQRs. (A) All Metabolites shared by GS and method. (B) Known (Named) Metabolites shared by GS and method.

between methods were observed for the Actinobacteria and Firmicutes (both are Gram-positive) than that for Bacteroidetes (Gram-negative), which is consistent with a previous study (Vogtmann et al., 2017a). This may partially explain the low concordance for Bacteroidetes between methods, but the exact mechanisms remain unclear. Taken together, method-related variations should be carefully considered if multiple fecal collection methods are used in the analyses, though all four methods reported here are generally comparable to a frozen sample for gut microbiome analyses.
This study also compared different fecal sample collection methods for untargeted metabolomics. Our analyses at multiple detectability levels indicated 95\% ethanol and FTA cards had comparable numbers of metabolites with high detectability when compared with immediate freezing. Particularly, among 132 known metabolites with $\geq 75 \%$ detectability in immediately frozen fecal samples, $91 \%$ and $77 \%$ of these metabolites were detected with $95 \%$ ethanol and FTA cards, respectively. However, OMNIgene GUT had many fewer detected metabolites. In a previous comparison study of untargeted metabolomics in fecal samples, 95\% ethanol also showed the largest number and highest concordance of overlapping metabolites with the frozen sample, followed by FOBT cards (Loftfield et al., 2016). Given the existence of better alternative methods, OMNIgene GUT might not be recommended as the collection method for untargeted metabolomics in fecal samples. Another point worth noting is that RNAlater was not feasible for metabolomics measures. This was likely due to the high sodium sulfate content in the samples collected with RNAlater, which made the collection incompatible with mass spectrometry-based metabolomics platforms (Loftfield et al., 2016; Sinha et al., 2016).

An important contribution of this study is that we specifically compared fecal collection methods for targeted metabolomics on SCFAs, a family of disease-related metabolites highly relevant to gut microbiota. Our data indicated that for the most predominant SCFAs (butyric acid and propionic acid) both OMNIgene GUT and FTA cards had high concordance (all ICCs $\geq 0.80$ ) with the immediate freezing method. For acetic acid, the ICCs were lower but still acceptable $(0.64,0.54)$. More importantly, we observed biologically plausible correlations between bacterial genera and the predominant SCFAs in fecal samples. Most correlations were reproduced with immediate freezing, OMNIgene GUT and FTA cards and the correlation coefficients were similar across these collection methods. For instance, we found that the genus Blautia was positively correlated with butyric acid using the frozen sample $(r=0.83)$ and this positive correlation was also observed by FTA cards $(r=0.74)$ and OMNIgene GUT $(r=0.62)$. This is in line with the fact that Blautia is a known butyrate producer (Berni Canani et al., 2016; Takahashi et al., 2016; Tanaka et al., 2016) and possesses the capability to produce butyric acid (Zhou et al., 2017). In addition, the negative correlation between Rikenellaceae and butyric acid was highly consistent across the frozen sample $(r=-0.71)$, OMNIgene GUT $(r=-0.94)$, and FTA cards $(r$ $=-0.83)$. Interestingly, this negative correlation has also been observed with rat fecal samples (Lin et al., 2016).

Besides a relatively small sample size, limitations of this study included the fact that participation was limited to healthy individuals. Future methodological work is need to ensure that results can be translated to other settings including patient groups and population-based epidemiological studies. Only one sample was collected for each method per individual in this study and we did not test reproducibility, although the reproducibility for these collection methods have been well-established. Finally, we did not examine the stability of each method over longterm storage. Many previous studies have reported relatively high stability of samples collected using FTA card or FOBT card, 95\% 


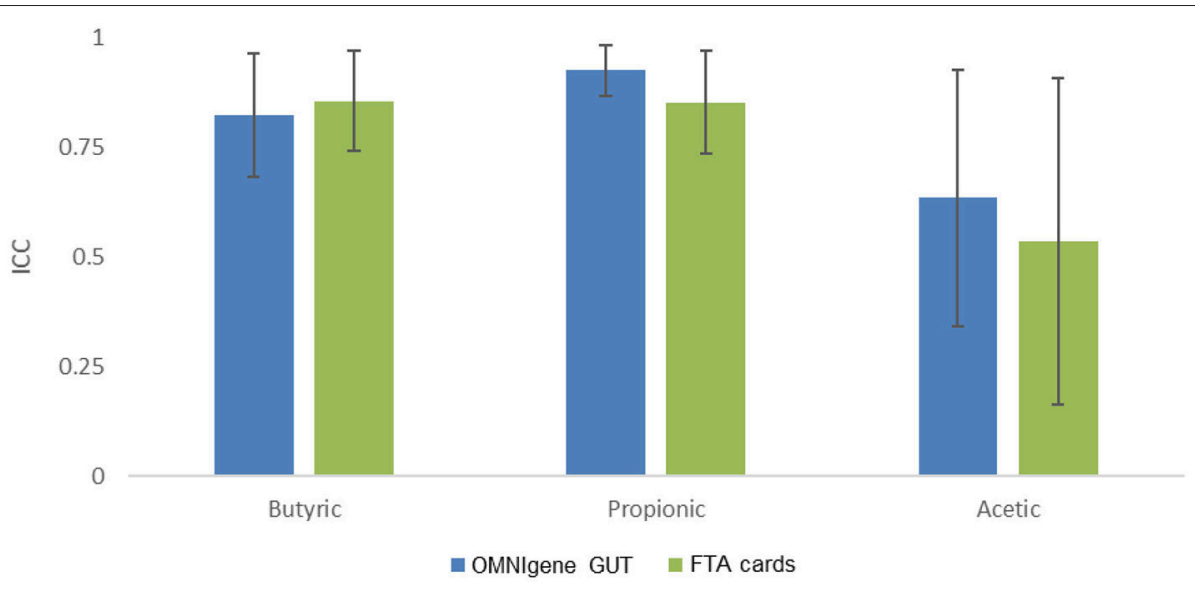

FIGURE 4 | The concordance of fecal collection methods compared with the gold standard immediate freezing: intraclass correlation coefficients (ICC) and 95\% Cl for the three predominant SCFAs.

TABLE 2 | Short chain fatty acids associated taxonomy: Genera which were significantly correlated with butyric acid, propionic acid and acetic acid, by Spearman correlation coefficients.

\begin{tabular}{|c|c|c|c|c|c|c|}
\hline & \multicolumn{2}{|c|}{ Immediate freezing } & \multicolumn{2}{|c|}{ OMNIgene GUT } & \multicolumn{2}{|c|}{ FTA cards } \\
\hline Blautia & 0.83 & 0.010 & 0.62 & 0.102 & 0.74 & 0.037 \\
\hline Rikenellaceae_UCG\# & -0.71 & 0.047 & -0.91 & 0.002 & -0.83 & 0.010 \\
\hline Shuttleworthia & -0.78 & 0.022 & -0.59 & 0.123 & 0.07 & 0.867 \\
\hline Clostridiales_UCG\# & -0.71 & 0.047 & -0.91 & 0.002 & -0.49 & 0.217 \\
\hline \multicolumn{7}{|l|}{ PROPIONIC ACID } \\
\hline Faecalibacterium & 0.88 & 0.004 & 0.45 & 0.260 & 0.74 & 0.037 \\
\hline Eubacterium & -0.76 & 0.029 & -0.41 & 0.320 & -0.31 & 0.453 \\
\hline \multicolumn{7}{|l|}{ ACETIC ACID } \\
\hline ML615J-28_UCG ${ }^{\#}$ & 0.75 & 0.033 & 0.17 & 0.694 & 0.38 & 0.359 \\
\hline
\end{tabular}

"P $<0.002$ (critical P-value after Bonferroni correction). "UCG: unclassified genus. RNAlater samples could not be run for this assay.

ethanol and RNAlater for microbial community analyses even preserved at ambient temperature for 4-14 days (Nechvatal et al., 2008; Franzosa et al., 2014; Flores et al., 2015; Voigt et al., 2015; Loftfield et al., 2016; Sinha et al., 2016; Song et al., 2016). Others have concluded that $95 \%$ ethanol and FOBT card collection methods were satisfactory for untargeted metabolomics analyses after 4 days, although not all methods tested in our study were evaluated in this prior study (Loftfield et al., 2016).

In summary, this study compared the concordance of the mainstream fecal collection technologies with the current gold standard method of immediate freezing for both microbiome and metabolomics analyses. The accuracy of FTA cards, OMNIgene GUT, and RNAlater were acceptable for microbiome analyses, though variations, especially in taxonomy, were observed across different collection methods, similar to the findings in previous methodological studies (Flores et al., 2015; Song et al., 2016; Vogtmann et al., 2017a). For untargeted metabolomics, the concordance was highest with $95 \%$ ethanol and acceptable with FTA cards. Furthermore, this study, for the first time, indicated that FTA cards and OMNIgene GUT are generally comparable with the gold standard immediate freezing sample for measurement of SCFAs and integrated analysis of gut microbiota and SCFAs. Given the costs and technical challenges of collecting stool samples that are immediately frozen by participants and transported with cold packs, our data document comparable alternative methods for large epidemiological studies.

\section{AUTHOR CONTRIBUTIONS}

ZW, RB and QQ analyzed data and wrote the manuscript. QQ, $\mathrm{RB}, \mathrm{RK}, \mathrm{HS}$, and CI designed the experiments of this study. CZ, $\mathrm{MU}$, and RB performed 16S rRNA gene sequencing experiments and $\mathrm{ZW}, \mathrm{MU}, \mathrm{RB}$, and QQ analyzed gut microbiota data. YQ and IK performed untargeted and targeted metabolomics 
experiments and $\mathrm{ZW}, \mathrm{QQ}$, YP, TW, and IK analyzed the metabolomics data.

\section{FUNDING}

This work was supported in part by National Heart, Lung, and Blood Institute (NHLBI) K01HL129892, R01HL140976, R01HL126543, R01HL132794, R01HL083760, and R01HL095140, Feldstein Medical Foundation Research Grant to QQ, R01MD011389, U01 AI035004, the Einstein

\section{REFERENCES}

Anders, S., and Huber, W. (2010). Differential expression analysis for sequence count data. Genome Biol. 11:R106. doi: 10.1186/gb-2010-11-10-r106

Bahl, M. I., Bergström, A., and Licht, T. R. (2012). Freezing fecal samples prior to DNA extraction affects the firmicutes to bacteroidetes ratio determined by downstream quantitative PCR analysis. FEMS Microbiol. Lett. 329, 193-197. doi: 10.1111/j.1574-6968.2012.02523.x

Berni Canani, R., Sangwan, N., Stefka, A. T., Nocerino, R., Paparo, L., Aitoro, R. et al. (2016). Lactobacillus rhamnosus GG-supplemented formula expands butyrate-producing bacterial strains in food allergic infants. ISME J. 10, 742-750. doi: 10.1038/ismej.2015.151

Bolstad, B. M., Irizarry, R. A., Astrand, M., and Speed, T. P. (2003). A comparison of normalization methods for high density oligonucleotide array data based on variance and bias. Bioinformatics 19, 185-193. doi: 10.1093/bioinformatics/19.2.185

Candela, M., Guidotti, M., Fabbri, A., Brigidi, P., Franceschi, C., and Fiorentini, C. (2011). Human intestinal microbiota: cross-talk with the host and its potential role in colorectal cancer. Crit. Rev. Microbiol. 37, 1-14. doi: 10.3109/1040841X.2010.501760

Caporaso, J. G., Bittinger, K., Bushman, F. D., DeSantis, T. Z., Andersen, G. L., and Knight, R. (2010a). PyNAST: a flexible tool for aligning sequences to a template alignment. Bioinformatics 26, 266-267. doi: 10.1093/bioinformatics/btp636

Caporaso, J. G., Kuczynski, J., Stombaugh, J., Bittinger, K., Bushman, F. D., Costello, E. K. et al. (2010b). QIIME allows analysis of highthroughput community sequencing data. Nat. Methods 7, 335-336. doi: $10.1038 /$ nmeth.f.303

Caporaso, J. G., Lauber, C. L., Walters, W. A., Berg-Lyons, D., Huntley, J., Fierer, N. et al. (2012). Ultra-high-throughput microbial community analysis on the illumina HiSeq and MiSeq platforms. ISME J. 6, 1621-1624. doi: 10.1038/ismej.2012.8

Cardona, S., Eck, A., Cassellas, M., Gallart, M., Alastrue, C., Dore, J. et al. (2012). Storage conditions of intestinal microbiota matter in metagenomic analysis. BMC Microbiol. 12:158. doi: 10.1186/1471-2180-12-158

Chong, J., and Xia, J. (2017). Computational approaches for integrative analysis of the metabolome and microbiome. Metabolites 7:E62. doi: 10.3390/metabo7040062

Choo, J. M., Leong, L. E., and Rogers, G. B. (2015). Sample storage conditions significantly influence faecal microbiome profiles. Sci. Rep. 5:16350. doi: $10.1038 /$ srep 16350

DeSantis, T. Z., Hugenholtz, P., Larsen, N., Rojas, M., Brodie, E. L., Keller, K. et al. (2006). Greengenes, a chimera-checked 16S rRNA gene database and workbench compatible with ARB. Appl. Environ. Microbiol. 72, 5069-5072. doi: 10.1128/AEM.03006-05

Dominianni, C., Wu, J., Hayes, R. B., and Ahn, J. (2014). Comparison of methods for fecal microbiome biospecimen collection. BMC Microbiol. 14:103. doi: 10.1186/1471-2180-14-103

Fiehn, O. (2002). Metabolomics-the link between genotypes and phenotypes. Plant Mol. Biol. 48, 155-171. doi: 10.1023/A:1013713905833

Flores, R., Shi, J., Gail, M. H., Gajer, P., Ravel, J., and Goedert, J. J. (2012). Assessment of the human faecal microbiota: II. reproducibility and associations of 16S rRNA pyrosequences. Eur. J. Clin. Investig. 42, 855-863. doi: 10.1111/j.1365-2362.2012.02659.x
Cancer Research Center (P30CA013330), the Einstein Liver Research Center (P30DK041296), and the Einstein-RockefellerCUNY Center for AIDS Research funded by the NIAID (P30AI124414).

\section{SUPPLEMENTARY MATERIAL}

The Supplementary Material for this article can be found online at: https://www.frontiersin.org/articles/10.3389/fcimb. 2018.00301/full\#supplementary-material
Flores, R., Shi, J., Yu, G., Ma, B., Ravel, J., Goedert, J. J. et al. (2015). Collection media and delayed freezing effects on microbial composition of human stool. Microbiome 3:33. doi: 10.1186/s40168-015-0092-7

Forslund, K., Hildebrand, F., Nielsen, T., Falony, G., Le Chatelier, E., Sunagawa, S., et al. (2015). Disentangling type 2 diabetes and metformin treatment signatures in the human gut microbiota. Nature 528, 262-266. doi: 10.1038/nature 15766

Fouhy, F., Deane, J., Rea, M. C., O’Sullivan, Ó., Ross, R. P., O’Callaghan, G. et al. (2015). The effects of freezing on faecal microbiota as determined using MiSeq sequencing and culture-based investigations. PLOS ONE 10:e0119355. doi: 10.1371/journal.pone.0119355

Franzosa, E. A., Morgan, X. C., Segata, N., Waldron, L., Reyes, J., Earl, A. M. et al. (2014). Relating the metatranscriptome and metagenome of the human gut. Proc. Natl. Acad. Sci. U.S.A. 111, E2329-E2338. doi: 10.1073/pnas.131 9284111

Kostic, A. D., Xavier, R. J., and Gevers, D. (2014). The microbiome in inflammatory bowel disease: current status and the future ahead. Gastroenterology 146, 1489-1499. doi: 10.1053/j.gastro.2014.02.009

Ley, R. E. (2010). Obesity and the human microbiome. Curr. Opin. Gastroenterol. 26, 5-11. doi: 10.1097/MOG.0b013e328333d751

Lin, H., An, Y., Hao, F., Wang, Y., and Tang, H. (2016). Correlations of fecal metabonomic and microbiomic changes induced by high-fat diet in the preobesity state. Sci. Rep. 6:21618. doi: 10.1038/srep21618

Loftfield, E., Vogtmann, E., Sampson, J. N., Moore, S. C., Nelson, H., Knight, R. et al. (2016). Comparison of collection methods for fecal samples for discovery metabolomics in epidemiologic studies. Cancer Epidemiol. Biomarkers Prev. 25, 1483-1490. doi: 10.1158/1055-9965.EPI-16-0409

Love, M. I., Huber, W., and Anders, S. (2014). Moderated estimation of fold change and dispersion for RNA-seq data with DESeq2. Genome Biol. 15:550. doi: 10.1186/s13059-014-0550-8

Magoc, T., and Salzberg, S. L. (2011). FLASH: fast length adjustment of short reads to improve genome assemblies. Bioinformatics 27, 2957-2963. doi: 10.1093/bioinformatics/btr507

Martin, F. P., Sprenger, N., Yap, I. K., Wang, Y., Bibiloni, R., Rochat, F. et al. (2009). Panorganismal gut microbiome-host metabolic crosstalk. J. Proteome Res. 8, 2090-2105. doi: 10.1021/pr801068x

McDonald, D., Price, M. N., Goodrich, J., Nawrocki, E. P., DeSantis, T. Z., Probst, A. et al.. (2012). An improved greengenes taxonomy with explicit ranks for ecological and evolutionary analyses of bacteria and archaea. ISME J. 6, 610-618. doi: 10.1038/ismej.2011.139

McMurdie, P. J., and Holmes, S. (2013). Phyloseq: an R package for reproducible interactive analysis and graphics of microbiome census data. PLOS ONE 8:e61217. doi: 10.1371/journal.pone.0061217

Nakatsu, G., Li, X., Zhou, H., Sheng, J., Wong, S. H., Wu, W. K. et al. (2015). Gut mucosal microbiome across stages of colorectal carcinogenesis. Nat. Commun. 6:8727. doi: $10.1038 /$ ncomms 9727

Nechvatal, J. M., Ram, J. L., Basson, M. D., Namprachan, P., Niec, S. R., Badsha, K. Z. et al. (2008). Fecal collection, ambient preservation, and DNA extraction for PCR amplification of bacterial and human markers from human feces. J. Microbiol. Methods 72, 124-132. doi: 10.1016/j.mimet.2007.11.007

Oksanen, J. (2015). Multivariate Analysis of Ecological Communities in R: Vegan Tutorial. Version 2.3-0. San Francisco, CA. Available online at: http://cc.oulu. fi/ jarioksa/opetus/metodi/vegantutor.pdf 
Patti, G. J., Yanes, O., and Siuzdak, G. (2012). Innovation: metabolomics: the apogee of the omics trilogy. Nat. Rev. Mol. Cell Biol. 13, 263-269. doi: $10.1038 / \mathrm{nrm} 3314$

Qiu, Y., Cai, G., Su, M., Chen, T., Zheng, X., Xu, Y. et al. (2009). Serum metabolite profiling of human colorectal cancer using GC-TOFMS and UPLC-QTOFMS. J. Proteome Res. 8, 4844-4850. doi: 10.1021/pr9004162

Schmieder, R., and Edwards, R. (2011). Quality control and preprocessing of metagenomic datasets. Bioinformatics 27, 863-864. doi: 10.1093/bioinformatics/btr026

Sinha, R., Abnet, C. C., White, O., Knight, R., and Huttenhower, C. (2015). The microbiome quality control project: baseline study design and future directions. Genome Biol. 16:276. doi: 10.1186/s13059-0150841-8

Sinha, R., Chen, J., Amir, A., Vogtmann, E., Shi, J., Inman, K. S. et al. (2016). Collecting fecal samples for microbiome analyses in epidemiology studies. Cancer Epidemiol. Biomarkers Prev. 25, 407-416. doi: 10.1158/1055-9965.EPI-15-0951

Song, S. J., Amir, A., Metcalf, J. L., Amato, K. R., Xu, Z. Z., Humphrey, G. et al. (2016). Preservation methods differ in fecal microbiome stability, affecting suitability for field studies. mSystems 1:e00021-16. doi: 10.1128/mSystems.00021-16

Takahashi, K., Nishida, A., Fujimoto, T., Fujii, M., Shioya, M., Imaeda, H. et al. (2016). Reduced abundance of butyrate-producing bacteria species in the fecal microbial community in crohn's disease. Digestion 93, 59-65. doi: $10.1159 / 000441768$

Tanaka, S., Yamamoto, K., Yamada, K., Furuya, K., and Uyeno, Y. (2016). Relationship of enhanced butyrate production by colonic butyrate-producing bacteria to immunomodulatory effects in normal mice fed an insoluble fraction of brassica rapa L. Appl. Environ. Microbiol. 82, 2693-2699. doi: 10.1128/AEM.03343-15

Vandeputte, D., Tito, R. Y., Vanleeuwen, R., Falony, G., and Raes, J. (2017). Practical considerations for large-scale gut microbiome studies. FEMS Microbiol. Rev. 41(Suppl. _1), S154-S167. doi: 10.1093/femsre/ fux 027
Vogtmann, E., Chen, J., Amir, A., Shi, J., Abnet, C. C., Nelson, H. et al. (2017b). Comparison of collection methods for fecal samples in microbiome studies. Am. J. Epidemiol. 185, 115-123. doi: 10.1093/aje/kww177

Vogtmann, E., Chen, J., Kibriya, M. G., Chen, Y., Islam, T., Eunes, M. et al. (2017a). Comparison of fecal collection methods for microbiota studies in bangladesh. Appl. Environ. Microbiol. 83:e00361-17. doi: 10.1128/AEM.00361-17

Vogtmann, E., and Goedert, J. J. (2016). Epidemiologic studies of the human microbiome and cancer. Br. J. Cancer 114, 237-242. doi: 10.1038/bjc.2015.465

Voigt, A. Y., Costea, P. I., Kultima, J. R., Li, S. S., Zeller, G., Sunagawa, S. et al. (2015). Temporal and technical variability of human gut metagenomes. Genome Biol. 16:73. doi: 10.1186/s13059-015-0639-8

Wong, W. S. W., Clemency, N., Klein, E., Provenzano, M., Iyer, R., Niederhuber, J. E. et al. (2017). Collection of non-meconium stool on fecal occult blood cards is an effective method for fecal microbiota studies in infants. Microbiome 5:114. doi: 10.1186/s40168-017-0333-Z

Zheng, X., Qiu, Y., Zhong, W., Baxter, S., Su, M., Li, Q., et al. Jia, W. (2013). A targeted metabolomic protocol for short-chain fatty acids and branched-chain amino acids. Metabolomics 9, 818-827. doi: 10.1007/s11306-013-0500-6

Zhou, D., Pan, Q., Xin, F. Z., Zhang, R. N., He, C. X., Chen, G. Y., et al. Fan, J. G. (2017). Sodium butyrate attenuates high-fat diet-induced steatohepatitis in mice by improving gut microbiota and gastrointestinal barrier. World J. Gastroenterol. 23, 60-75. doi: 10.3748/wjg.v23.i1.60

Conflict of Interest Statement: The authors declare that the research was conducted in the absence of any commercial or financial relationships that could be construed as a potential conflict of interest.

Copyright (c) 2018 Wang, Zolnik, Qiu, Usyk, Wang, Strickler, Isasi, Kaplan, Kurland, Qi and Burk. This is an open-access article distributed under the terms of the Creative Commons Attribution License (CC BY). The use, distribution or reproduction in other forums is permitted, provided the original author(s) and the copyright owner(s) are credited and that the original publication in this journal is cited, in accordance with accepted academic practice. No use, distribution or reproduction is permitted which does not comply with these terms. 